\title{
Utility of Genomic Testing after Renal Biopsy
}

\author{
Susan L. Murray ${ }^{a, g}$ Anthony Dorman ${ }^{b, c}$ Katherine A. Benson ${ }^{e}$ \\ Dervla M. Connaughton ${ }^{a, d}$ Caragh P. Stapleton ${ }^{\mathrm{e}}$ Neil K. Fennelly ${ }^{\mathrm{b}}$ \\ Claire Kennedy $^{a}$ Ciara A. McDonnella Kendrah Kidd ${ }^{f}$ Sarah M. Cormican $^{a}$ \\ Louise A. Ryan ${ }^{a}$ Peter Lavin ${ }^{h}$ Mark A. Little Anthony J. Bleyer $^{f}$ Brendan Doyle $^{b}$ \\ Gianpiero L. Cavallerie Friedhelm Hildebrandt ${ }^{d}$ Peter J. Conlon ${ }^{\mathrm{a}} \mathrm{g}$ \\ ${ }^{a}$ Department of Nephrology and Transplantation, Beaumont Hospital, Dublin, Ireland; ${ }^{b}$ Department of Pathology, \\ Beaumont Hospital, Dublin, Ireland; ' Department of Pathology, Royal College of Surgeons in Ireland, Dublin, Ireland; \\ ${ }^{\mathrm{d}}$ Department of Medicine, Boston Children's Hospital, Harvard Medical School, Boston, MA, USA; ${ }^{\text {eDepartment of }}$

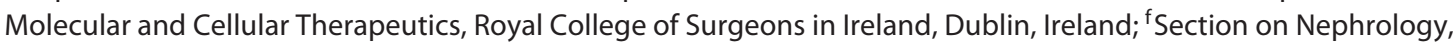 \\ Wake Forest School of Medicine, Winston-Salem, NC, USA; ${ }^{9}$ Department of Medicine, Royal College of Surgeons in \\ Ireland, Dublin, Ireland; ' Department of Nephrology, Tallaght Hospital, Dublin, Ireland; iTrinity Health Kidney Centre, \\ Trinity Translational Medicine Institute, Trinity College Dublin, Dublin, Ireland
}

\section{Keywords}

Renal biopsy · Pathology · Chronic kidney disease ·

Genetics · Genetic polymorphism

\begin{abstract}
Background: Renal biopsy is the mainstay of renal pathological diagnosis. Despite sophisticated diagnostic techniques, it is not always possible to make a precise pathological diagnosis. Our aim was to identify a genetic cause of disease in patients who had undergone renal biopsy and determine if genetic testing altered diagnosis or treatment. Methods: Patients with suspected familial kidney disease underwent a variety of next-generation sequencing (NGS) strategies. The subset of these patients who had also undergone native kidney biopsy was identified. Histological specimens were reviewed by a consultant pathologist, and genetic and pathological diagnoses were compared. Results: Seventy-five patients in 47 families underwent genetic se-
\end{abstract}

quencing and renal biopsy. Patients were grouped into 5 diagnostic categories based on pathological diagnosis: tubulointerstitial kidney disease (TIKD; $n=18$ ); glomerulonephritis (GN; $n=15)$; focal segmental glomerulosclerosis and Alport Syndrome ( $n=11)$; thrombotic microangiopathy (TMA; $n=17)$; and nonspecific pathological changes $(n=14)$. Thirty-nine patients (52\%) in 21 families (45\%) received a genetic diagnosis; 13 cases (72\%) with TIKD, 4 (27\%) with GN, 6 (55\%) with focal segmental glomerulosclerosis/Alport syndrome, and 10 (59\%) with TMA and 6 cases (43\%) with nonspecific features. Genetic testing resulted in changes in understanding of disease mechanism in 21 individuals (54\%) in 12 families (57\%). Treatment would have been altered in at least $26 \%$ of cases (10/39). Conclusions: An accurate genetic diagnosis can result in changes in clinical diagnosis, understanding of pathological mechanism, and treatment. NGS should be considered as a complementary diagnostic technique to kidney biopsy in the evaluation of patients with kidney disease.

(c) 2019 S. Karger AG, Basel

\section{KARGER}

(c) 2019 S. Karger AG, Basel

E-Mail karger@karger.com

www.karger.com/ajn
Susan L. Murray

Department of Nephrology and Transplantation

Beaumont Hospital

Dublin 9 (Ireland)

E-Mail susanmurray@beaumont.ie 


\section{Introduction}

As a procedure, the percutaneous renal biopsy is nearly 70 years old. Since it was first described by Iversen and Braun [1], kidney biopsy has become the gold standard for renal pathological diagnosis [2]. Light microscopy, immunofluorescence, and electron microscopy have been refined over time to provide increasingly precise classification of kidney disease pathology. Standardized classifications guide therapy and define objective endpoints for treatment $[3,4]$.

Kidney biopsy is a safe procedure with a high diagnostic yield. It gives useful clinical information in $80 \%$ of cases $[5,6]$. A prospective study of 80 patients by Turner et al. [7] showed that renal biopsy modified diagnosis in $44 \%$ and therapeutic approach in $31 \%$ of patients. Other studies have shown that treatment is modified in up to $54 \%$ of patients [8].

Despite its utility as a therapeutic tool, pathological findings from renal biopsies are not completely accurate or precise. Even with the implementation of international guidelines, a significant degree of interobserver variability continues to exist [9]. Interobserver agreement is as low as $45 \%$ in some reports [10]. Alone, renal biopsy may be inadequate to distinguish different phenotypes of kidney disease and provide a precise diagnosis. Approximately $15 \%$ of all incident patients in the United Kingdom who reach end-stage renal disease (ESRD) do not have a primary renal diagnosis [11].

Next-generation sequencing (NGS) technology and associated diagnostic techniques have led to a reclassification of the etiology of many forms of kidney disease. There are now $>600$ genes known to harbor variants that are associated with kidney disease [12]. A recent study showed that whole exome sequencing can yield a genetic diagnosis in nearly $10 \%$ of patients with chronic kidney disease (CKD), including $17 \%$ of those with nephropathy of unknown origin [12].

The addition of molecular techniques to kidney biopsy as a diagnostic modality may improve precision and lead to more refined diagnosis, more reliable predictions of prognosis and a wider choice of therapeutic options. It may give better diagnostic certainty for patients and families and facilitates screening and genetic counseling. This may offer direct benefits in terms of an earlier diagnosis, and screening of potential living related renal donors who are twice as likely to develop ESRD as unrelated kidney donors [13].

The Irish Kidney Gene Project was established in 2015 to define the prevalence of a positive family history in a cohort of adult patients with CKD in Ireland and to apply NGS techniques to determine genetic causes of kidney disease in this cohort. Our aim was to identify the genetic cause of kidney disease in a cohort of patients who had previously undergone percutaneous kidney biopsy and to review the initial pathological diagnosis in light of this new information. We aimed to determine if genetic diagnosis would lead to a change in understanding of disease mechanism and if this changed understanding of disease mechanism would have implications for the treatment plan.

\section{Methods}

\section{Patient Population}

Participants were recruited from patients who attended nephrology services in Ireland from January 2014 to December 2017. Informed consent was obtained from all patients. The study was approved by the medical ethics board at the recruitment sites.

Patients were included if they were aged $>18$ years, capable of giving consent and had either a self-reported family history of CKD, or extrarenal features consistent with an inherited cause of kidney disease as adjudged by the treating nephrologist. They were excluded if they had not undergone percutaneous native renal biopsy. Demographic and clinical information and family history was obtained from participants. DNA was extracted from blood or saliva samples.

\section{Genetic Diagnosis}

A specific genetic diagnosis was obtained by NGS via one of the following 3 methods. Some samples were tested using multiple techniques:

1. In the first cohort of 138 participants, whole exome sequencing was performed in Boston Children's Hospital, Massachusetts, as previously described by Connaughton et al. [14].

2. A second cohort consisted of 54 individuals with autosomal dominant tubulointerstitial kidney disease (ADTKD) who were suspected of having ADTKD-MUC1 or ADTKD-UMOD. Gene testing for $M U C 1 \mathrm{C}+$ insertions was performed at the Broad Institute, Massachusetts, using techniques described elsewhere [15]. UMOD mutational analysis was performed in all UMOD exons by the Rare Inherited Kidney Disease team of Wake Forest School of Medicine, Winston-Salem, NC [16, 17].

3. A subsequent third cohort of 44 patients was sequenced using targeted NGS. Samples were sequenced in the Royal College of Surgeons in Ireland by targeted NGS using a custom Roche NimbleGen SeqCap or a Roche NimbleGen HeatSeq panel (genes listed in online suppl. Table 1; for all online suppl. material, see www.karger.com/doi/10.1159/000504869) as per the manufacturer's instructions, using $500 \mathrm{ng}$ of input gDNA. Sequencing was performed on an Illumina MiSeq or NextSeq. Sequence data were analyzed using a custom, in-house pipeline. Sequence data were aligned to the NCBI 138/hg38 reference genome and processed using a Burrows-Wheeler Aligner and Picard. Variants were identified using the Genome Analysis ToolKitbest practices protocoland annotated using ANNOVAR.
Murray et al. 
Table 1. Clinical characteristics of 76 individuals who underwent NGS and kidney biopsy

\begin{tabular}{|c|c|c|c|c|}
\hline Age at biopsy, years, median (range) & $36(7-69)$ & $33(10-61)$ & $38(7-69)$ & 0.11 \\
\hline Gender, male, $n(\%)$ & $49(65)$ & $26(66)$ & $27(75)$ & 0.3 \\
\hline Family history, $n(\%)$ & $69(92)$ & $37(95)$ & $32(89)$ & 0.33 \\
\hline GN & $15(20)$ & $4(10)$ & $11(31)$ & \\
\hline FSGS/Alport & $11(15)$ & $6(15.5)$ & $5(14)$ & \\
\hline TMA & $17(23)$ & $10(26)$ & $7(19)$ & \\
\hline Nonspecific features & $14(18)$ & $6(15.5)$ & $8(22)$ & \\
\hline Creatinine at biopsy, $\mu \mathrm{mol} / \mathrm{L}$, median (IQR) & $153(101-208)$ & $154(99-201)$ & $154(112-258)$ & 0.88 \\
\hline Developed ESRD & $52(69)$ & $28(72)$ & $24(66)$ & 0.63 \\
\hline
\end{tabular}

NGS, next-generation sequencing; TIKD, tubulointerstitial kidney disease; TMA, thrombotic microangiopathy; IQR, interquartile range; ESRD, end-stage renal disease; GN, glomerulonephritis.

Sequences with a minimum coverage of $\geq 10 \times$ were included for analysis. Rare variants (minor allele frequency $<0.01$ (homozygotes/compound heterozygotes) or minor allele frequency < 0.001 (heterozygotes) in gnom AD control database), functional (exonic/splicing variant), predicted damaging by at least 2 prediction software tools, and in a relevant disease gene (as per Online Mendelian Inheritance in Man) were selected for discussion at a multidisciplinary team meeting.

In all cases, potentially causative variants were classified as pathogenic, likely pathogenic, a variant of unknown significance, likely benign or benign as per the guidelines of the American College of Medical Genetics [18].

\section{Pathological Diagnosis}

We identified all sequenced patients who had undergone a renal biopsy. Biopsies were reviewed independently by an experienced renal histopathologist (A.D.) in Beaumont Hospital, Dublin (online suppl. Table 2). Where available, electron micrographs were also reviewed. The histopathologist reassessed the histological slides and compared them to the original results. If there was a discrepancy between the 2 , the diagnosis was changed to reflect the diagnosis on re-assessment. The histopathologist was blinded to the gene sequencing results. Where review could not be performed due to inadequate condition or suitability, the original pathological diagnosis was used. Original slides were available and in acceptable condition in $92 \%$ of all cases. Electron microscopy was available in $79 \%$ of cases.

The medical and histological diagnosis of all patients were reviewed and recorded, including glomerular, interstitial, vascular, and tubular features as well as percentage fibrosis.

Following review of biopsy material, renal pathological diagnosis was divided into 5 categories:

- Tubulointerstitial kidney disease (TIKD)

- Chronic glomerulonephritis (GN)
- Focal segmental glomerulosclerosis (FSGS) and Alport syndrome

- Thrombotic microangiopathy (TMA)

- Nonspecific pattern of injury

\section{Statistical Analysis}

Descriptive statistics were expressed using frequencies and proportions.

Unpaired $t$ tests and chi-square were used to test for significance between those in whom a genetic diagnosis was obtained and those in whom one was not obtained. A $p$ value of $<0.05$ was considered statistically significant.

\section{Results}

A total of 75 individuals in 47 families had undergone renal biopsy and genetic testing. Of those 75 patients, a pathogenic or likely pathogenic, disease-causing variant that met American College of Medical Genetics criteria (online suppl. Table 3) was detected in 39 cases (52\%) in 21 families (45\%). In the remaining 36 patients (48\%) and 26 families (55\%), we were unable to identify a pathogenic variant. A family history was present in 69 patients (92\%).

The mean age of patients at the time of renal biopsy was 36 years and $65 \%$ were male. There were no statistical differences in age at biopsy, sex, risk of progressing to ESRD, creatinine at biopsy, or presence of a family history between those who obtained a genetic diagnosis and those that did not (Table 1). The median time from 
Table 2. Information on genetic diagnosis in 75 individuals who underwent NGS and histological diagnosis by renal pathological diagnostic group

\begin{tabular}{|c|c|c|}
\hline Pathological diagnosis & Genetic diagnosis & Number affected, $n(\%)$ \\
\hline $\operatorname{TIKD}(n=18)$ & $\begin{array}{l}\text { MUC1 } \\
\text { UMOD } \\
\text { HNF1B } \\
\text { NPHP } 1 \\
\text { IFT140 } \\
\text { No diagnosis }\end{array}$ & $\begin{array}{l}6(34) \\
4(22) \\
1(5.5) \\
1(5.5) \\
1(5.5) \\
5(27.5)\end{array}$ \\
\hline Chronic GN $(n=15)$ & $\begin{array}{l}\text { COL4A5 } \\
\text { UMOD } \\
\text { MUC1 } \\
\text { No diagnosis }\end{array}$ & $\begin{array}{c}2(13) \\
1(7) \\
1(7) \\
11(73)\end{array}$ \\
\hline $\begin{array}{l}\text { Focal segmental glomerulosclerosis/ } \\
\text { Alport Syndrome }(n=11)\end{array}$ & $\begin{array}{l}\text { COL4A5 } \\
\text { FANCI } \\
\text { No diagnosis }\end{array}$ & $\begin{array}{l}5(45) \\
1(10) \\
5(45)\end{array}$ \\
\hline TMA $(n=17)$ & $\begin{array}{l}\text { UMOD } \\
\text { HNF1B } \\
\text { MUC1 } \\
\text { INF2 } \\
\text { IFT140 } \\
\text { No diagnosis }\end{array}$ & $\begin{array}{l}2(11.5) \\
2(11.5) \\
1(6) \\
4(24) \\
1(6) \\
7(41)\end{array}$ \\
\hline Nonspecific causes $(n=14)$ & $\begin{array}{l}\text { COL4A5 } \\
\text { C3 } \\
\text { WNK4 } \\
\text { SLC3A1 } \\
\text { HNF1B } \\
\text { INF2 } \\
\text { No diagnosis }\end{array}$ & $\begin{array}{l}1(7) \\
1(7) \\
1(7) \\
1(7) \\
1(7) \\
1(7) \\
8(58)\end{array}$ \\
\hline
\end{tabular}

NGS, next-generation sequencing; TIKD, tubulo-interstitial kidney disease; TMA, thrombotic microangiopathy; GN, glomerulonephritis.

biopsy to genetic diagnosis was 15 years (range 1-46 years).

Following review of the pathological diagnosis, TIKD accounted for the histological diagnosis in 18 cases (24\%) and 6 families (13\%), chronic GN in 15 patients (20\%) and 8 families (17\%), FSGS and Alport Syndrome in 11 cases (15\%) and 10 families (21\%), TMA in 17 cases (23\%) and 4 families (9\%), and nonspecific findings in 14 patients $(18 \%)$ or 11 families (23\%; Table 2). In the additional 8 families (17\%), there was a conflicting pathological diagnosis between 2 or more family members. Six of these families had at least 1 family member whose biopsy showed TMA.

Of the 39 patients in whom a genetic diagnosis was made, the genetic diagnosis was provided by testing in cohort 1 in 13 patients (33\%) and had been previously reported by Connaughton et al. [14]. The diagnostic rate in this cohort was 39\%. Cohort 2 provided diagnosis in 13 (33\%) of all patients. Diagnostic rate was $72 \%$. Cohort 3 provided a genetic diagnosis in 13 patients (33\%). Diagnostic rate was $52 \%$.

In the 18 patients with a preexisting pathological diagnosis of TIKD, a genetic diagnosis was made in 13 cases (72\%; MUC1, $n=6$; UMOD, $n=4$; HNF1B, $n=1$; IFT140, $n=1 ; N P H P 1, n=1$ ) and 6 families (Table 3). In all 13 cases, there was concordance between the a priori histological subtype and the genetic diagnosis. In 3 families, the diagnosis confirmed a suspected clinical and pathological diagnosis (ADTKD-MUC1, ADTKD-UMOD). In 1 family, it helped confirm the cause of extrarenal features (IFT140 causing Mainzer-Saldino syndrome) in a case of suspected nephronophthisis, in 2 further families (NPHP1 $\& H N F 1 B)$ it helped to identify a diagnosis in patients that had previously only been identified as nonspecific TIKD 


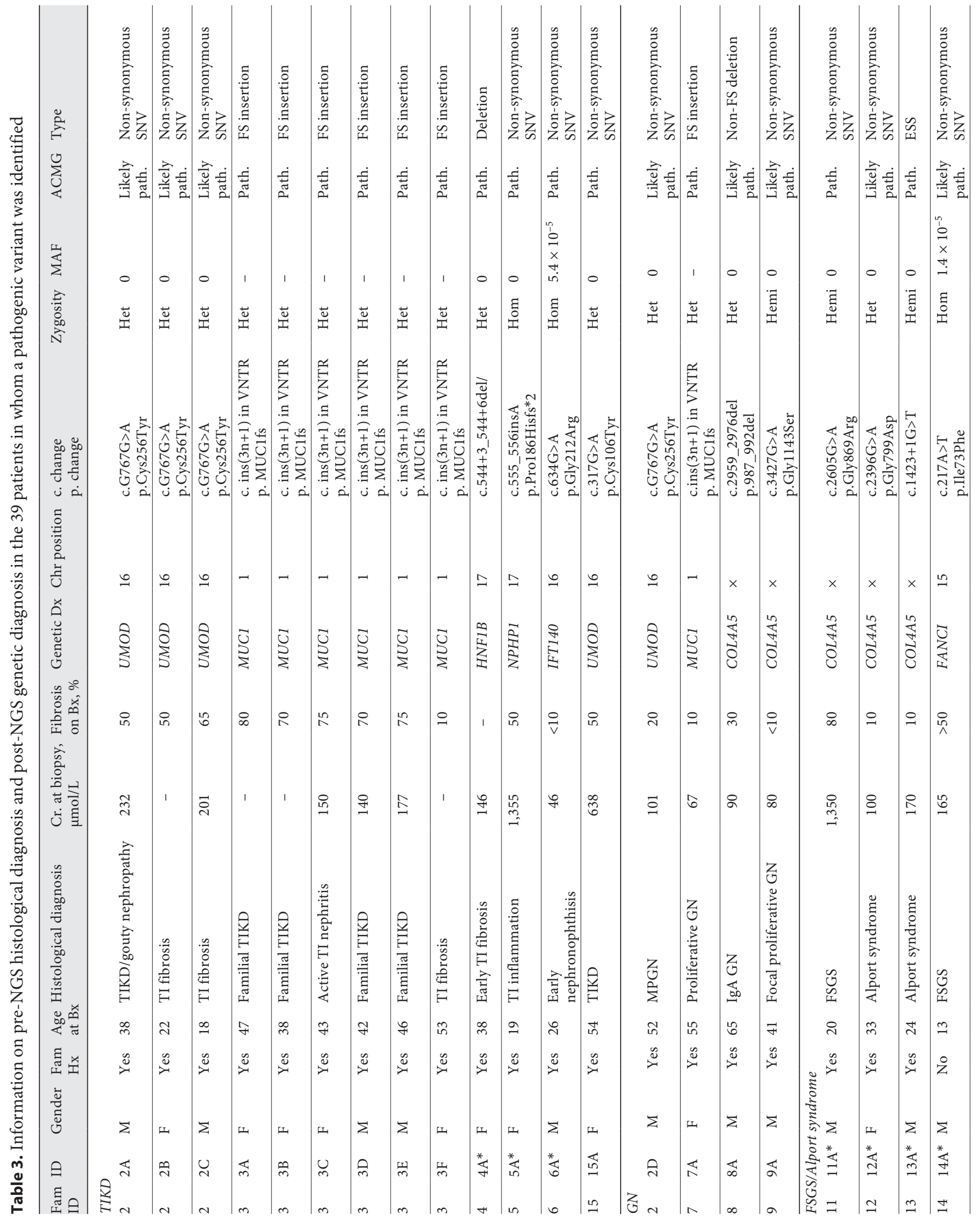




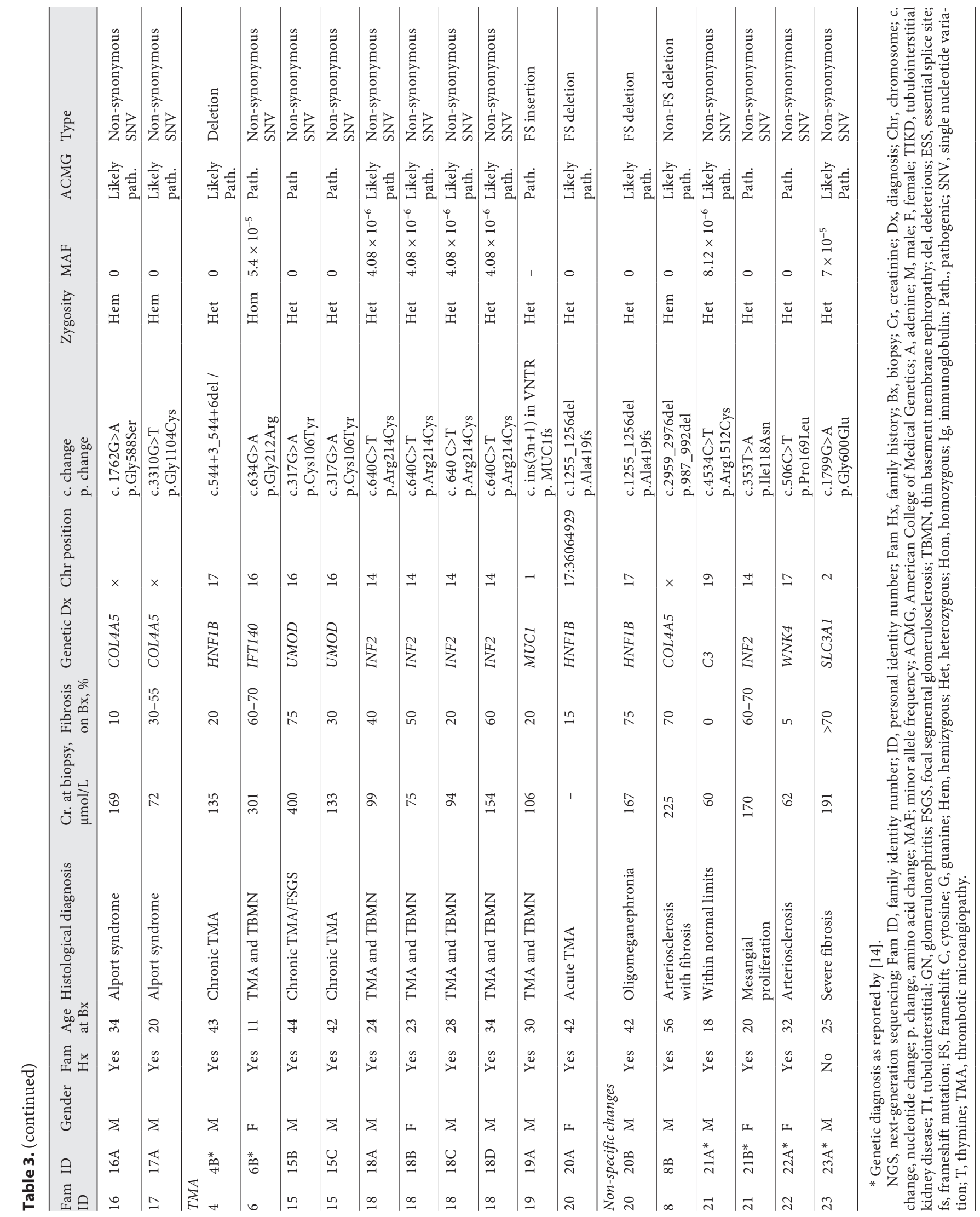


Table 4. Information on phenotype and histological diagnosis among families and family members, alteration to final diagnosis, and potential alterations to treatment following next generation sequencing

\begin{tabular}{|c|c|c|c|c|c|c|c|c|c|c|}
\hline $\begin{array}{l}\text { Family } \\
\text { ID }\end{array}$ & $\begin{array}{l}\text { Number of } \\
\text { affected } \\
\text { individuals }\end{array}$ & ID & Phenotype & $\begin{array}{l}\text { Histological } \\
\text { diagnosis }\end{array}$ & $\begin{array}{l}\text { Potential } \\
\text { change in } \\
\text { diagnosis }\end{array}$ & $\begin{array}{l}\text { Genetic } \\
\text { diagnosis }\end{array}$ & $\begin{array}{l}\text { Final diagnosis } \\
\text { (OMIM phenotype } \\
\text { MIM no.) }\end{array}$ & $\begin{array}{l}\text { Material } \\
\text { change in } \\
\text { diagnosis }\end{array}$ & $\begin{array}{l}\text { Potential } \\
\text { treatment } \\
\text { change }\end{array}$ & $\begin{array}{l}\text { Nature of } \\
\text { change }\end{array}$ \\
\hline \multirow{3}{*}{2} & \multirow{3}{*}{4} & $2 \mathrm{~B}$ & $\begin{array}{l}\text { Progressive CKD, } \\
\text { onset in 20s and early } \\
\text { onset gout }\end{array}$ & TI fibrosis & Yes & $U M O D$ & $\begin{array}{l}\text { ADTKD-UMOD } \\
(603860)\end{array}$ & No & No & \\
\hline & & $2 \mathrm{C}$ & $\begin{array}{l}\text { Progressive CKD, } \\
\text { onset in 20s and early } \\
\text { onset gout }\end{array}$ & TI fibrosis & Yes & $U M O D$ & $\begin{array}{l}\text { ADTKD-UMOD } \\
(603860)\end{array}$ & No & No & \\
\hline & & $2 \mathrm{D}$ & $\begin{array}{l}\text { Progressive CKD, } \\
\text { onset in 20s and early } \\
\text { onset gout }\end{array}$ & MPGN/DDD & Yes & $U M O D$ & $\begin{array}{l}\text { ADTKD-UMOD } \\
(603860)\end{array}$ & No & No & \\
\hline \multirow{4}{*}{3} & \multirow{4}{*}{6} & $3 \mathrm{~B}$ & $\begin{array}{l}\text { Progressive } \\
\text { non-proteinuric CKD } \\
\text { detected age } 38\end{array}$ & Familial TIKD & Yes & MUC1 & $\begin{array}{l}\text { ADTKD-MUC1 } \\
(174000)\end{array}$ & No & No & \\
\hline & & $3 \mathrm{C}$ & $\begin{array}{l}\text { Progressive } \\
\text { non-proteinuric CKD } \\
\text { detected mid-30s }\end{array}$ & Acute TI fibrosis & Yes & MUC1 & $\begin{array}{l}\text { ADTKD-MUC1 } \\
(174000)\end{array}$ & No & No & \\
\hline & & $3 \mathrm{D}$ & $\begin{array}{l}\text { Progressive } \\
\text { non-proteinuric CKD } \\
\text { detected mid-30s }\end{array}$ & Familial TIKD & Yes & MUC1 & $\begin{array}{l}\text { ADTKD-MUC1 } \\
(174000)\end{array}$ & No & No & \\
\hline & & $3 \mathrm{E}$ & $\begin{array}{l}\text { Progressive } \\
\text { non-proteinuric CKD } \\
\text { age } 40\end{array}$ & Familial TIKD & Yes & $M U C 1$ & $\begin{array}{l}\text { ADTKD-MUC1 } \\
(174000)\end{array}$ & No & No & \\
\hline 4 & 2 & $4 \mathrm{~B}^{*}$ & $\begin{array}{l}\text { CKD age } 42 \text {, diabetes } \\
\text { mellitus }\end{array}$ & TMA & Yes & $H N F 1 B$ & $\begin{array}{l}\text { ADTKD-HNF1B } \\
(137920)\end{array}$ & No & Yes & $\begin{array}{l}\text { Liver and } \\
\text { parathyroid } \\
\text { screening }\end{array}$ \\
\hline 5 & 1 & $5 A^{*}$ & $\begin{array}{l}\text { CKD, age } 21, \text { small } \\
\text { cystic kidneys on } \\
\text { renal US }\end{array}$ & TI nephritis & Yes & NPHP1 & $\begin{array}{l}\text { Nephronophthisis } 1 \text {, } \\
\text { juvenile } \\
(256100)\end{array}$ & No & No & \\
\hline \multirow[t]{2}{*}{6} & \multirow[t]{2}{*}{2} & $6 \mathrm{~A}^{*}$ & $\begin{array}{l}\text { Small cystic kidneys, } \\
\text { retinitis pigmentosa, } \\
\text { mild learning } \\
\text { disability }\end{array}$ & $\begin{array}{l}\text { Early } \\
\text { nephronophthisis }\end{array}$ & Yes & IFT140 & $\begin{array}{l}\text { Mainzer-Saldino } \\
\text { syndrome } \\
(266920)\end{array}$ & No & No & \\
\hline & & $6 \mathrm{~B}^{*}$ & $\begin{array}{l}\text { Small cystic kidneys, } \\
\text { retinitis pigmentosa, } \\
\text { mild learning } \\
\text { disability }\end{array}$ & TMA and TIKD & Yes & IFT140 & $\begin{array}{l}\text { Mainzer-Saldino } \\
\text { syndrome } \\
(266920)\end{array}$ & No & No & \\
\hline 7 & 1 & $7 \mathrm{~A}$ & $\begin{array}{l}\text { Low complement } \\
\text { (C3), gout, } \\
\text { arthropathy, family } \\
\text { history }\end{array}$ & $\begin{array}{l}\text { Proliferative } \\
\text { glomerulonephritis }\end{array}$ & Yes & MUC1 & $\begin{array}{l}\text { ADTKD-MUC1 } \\
(174000)\end{array}$ & Yes & Yes & $\begin{array}{l}\text { Steroid } \\
\text { avoidance }\end{array}$ \\
\hline
\end{tabular}


Table 4. (continued)

\begin{tabular}{|c|c|c|c|c|c|c|c|c|c|c|}
\hline $\begin{array}{l}\text { Family } \\
\text { ID }\end{array}$ & $\begin{array}{l}\text { Number of } \\
\text { affected } \\
\text { individuals }\end{array}$ & ID & Phenotype & $\begin{array}{l}\text { Histological } \\
\text { diagnosis }\end{array}$ & $\begin{array}{l}\text { Potential } \\
\text { change in } \\
\text { diagnosis }\end{array}$ & $\begin{array}{l}\text { Genetic } \\
\text { diagnosis }\end{array}$ & $\begin{array}{l}\text { Final diagnosis } \\
\text { (OMIM phenotype } \\
\text { MIM no.) }\end{array}$ & $\begin{array}{l}\text { Material } \\
\text { change in } \\
\text { diagnosis }\end{array}$ & $\begin{array}{l}\text { Potential } \\
\text { treatment } \\
\text { change }\end{array}$ & $\begin{array}{l}\text { Nature of } \\
\text { change }\end{array}$ \\
\hline 8 & & $8 \mathrm{~B}$ & $\begin{array}{l}\text { Progressive CKD } \\
\text { detected in } 40 \text { s, } \\
\text { hematuria detected } \\
\text { in } 20 \text { s }\end{array}$ & $\begin{array}{l}\text { Arteriosclerosis } \\
\text { with fibrosis }\end{array}$ & Yes & COL4A5 & $\begin{array}{l}\text { Alport syndrome I, } \\
\text { X linked } \\
(301050)\end{array}$ & Yes & Yes & $\begin{array}{l}\text { ENT and } \\
\text { ophthalmology } \\
\text { review }\end{array}$ \\
\hline 9 & 1 & $9 \mathrm{~A}$ & $\begin{array}{l}\text { Hypertension, } \\
\text { proteinuria and } \\
\text { haematuria }\end{array}$ & Focal proliferative GN & Yes & COL4A5 & $\begin{array}{l}\text { Alport syndrome I, } \\
\text { X linked } \\
(301050)\end{array}$ & Yes & Yes & $\begin{array}{l}\text { ENT and } \\
\text { ophthalmology } \\
\text { review }\end{array}$ \\
\hline 11 & 1 & $11 \mathrm{~A}$ & $\begin{array}{l}\text { Progressive CKD, } \\
\text { glaucoma and hearing } \\
\text { impairment }\end{array}$ & FSGS & Yes & COL4A5 & $\begin{array}{l}\text { Alport syndrome I, } \\
\text { X linked } \\
(301050)\end{array}$ & Yes & No & \\
\hline 13 & 1 & $13 A^{*}$ & $\begin{array}{l}\text { Progressive } \\
\text { hematuria, CKD and } \\
\text { hearing loss }\end{array}$ & Alport syndrome & No & COL4A5 & $\begin{array}{l}\text { Alport syndrome I, } \\
\text { X linked } \\
(301050)\end{array}$ & No & No & \\
\hline 14 & 1 & $14 \mathrm{~A}^{*}$ & $\begin{array}{l}\text { Bilateral small } \\
\text { kidneys, gout, retinitis } \\
\text { pigmentosa, anemia } \\
\text { and pseudotumor } \\
\text { cerebri }\end{array}$ & FSGS & Yes & FANCI & $\begin{array}{l}\text { Fanconi Anaemia, } \\
\text { complementation } \\
\text { group I } \\
(609053)\end{array}$ & Yes & Yes & $\begin{array}{l}\text { Cancer } \\
\text { screening }\end{array}$ \\
\hline 15 & 3 & $15 \mathrm{~A}$ & Progressive CKD & TIKD & Yes & $U M O D$ & $\begin{array}{l}\text { ADTKD-UMOD } \\
(603860)\end{array}$ & Yes & No & \\
\hline 17 & 1 & $17 \mathrm{~A}$ & $\begin{array}{l}\text { Hematuria, } \\
\text { progressive CKD } \\
\text { and hearing loss }\end{array}$ & Alport syndrome & No & COL4A5 & $\begin{array}{l}\text { Alport syndrome I, } \\
\text { X linked } \\
(301050)\end{array}$ & No & No & \\
\hline \multirow[t]{4}{*}{18} & 4 & $18 \mathrm{~A}$ & $\begin{array}{l}\text { Progressive CKD, } 1.8 \\
\text { g proteinuria, no } \\
\text { evidence of systemic } \\
\text { TMA }\end{array}$ & TMA and TBMN & Yes & $I N F 2$ & $\begin{array}{l}\text { Glomerulosclerosis, } \\
\text { focal segmental, } 5 \\
\text { (613237) }\end{array}$ & Yes & No & \\
\hline & & $18 \mathrm{~B}$ & $\begin{array}{l}\text { Proteinuria but normal } \\
\text { renal function, age } 42 \text {, } \\
\text { no evidence of systemic } \\
\text { TMA }\end{array}$ & TMA and TBMN & Yes & INF2 & $\begin{array}{l}\text { Glomerulosclerosis, } \\
\text { focal segmental, } 5 \\
\text { (613237) }\end{array}$ & Yes & No & \\
\hline & & $18 \mathrm{C}$ & $\begin{array}{l}\text { Proteinuria, } \\
\text { progressive CKD, no } \\
\text { evidence of systemic } \\
\text { TMA }\end{array}$ & TMA and TBMN & Yes & INF2 & $\begin{array}{l}\text { Glomerulosclerosis, } \\
\text { focal segmental, } 5 \\
(613237)\end{array}$ & Yes & No & \\
\hline & & $18 \mathrm{D}$ & $\begin{array}{l}\text { Progressive CKD, } \\
\text { ESRD age } 36, \text { no } \\
\text { evidence of systemic } \\
\text { TMA }\end{array}$ & TMA and TBMN & Yes & INF2 & $\begin{array}{l}\text { Glomerulosclerosis, } \\
\text { focal segmental, } 5 \\
\text { (613237) }\end{array}$ & Yes & No & \\
\hline
\end{tabular}


Table 4. (continued)

\begin{tabular}{|c|c|c|c|c|c|c|c|c|c|c|}
\hline $\begin{array}{l}\text { Family } \\
\text { ID }\end{array}$ & $\begin{array}{l}\text { Number of } \\
\text { affected } \\
\text { individuals }\end{array}$ & ID & Phenotype & $\begin{array}{l}\text { Histological } \\
\text { diagnosis }\end{array}$ & $\begin{array}{l}\text { Potential } \\
\text { change in } \\
\text { diagnosis }\end{array}$ & $\begin{array}{l}\text { Genetic } \\
\text { diagnosis }\end{array}$ & $\begin{array}{l}\text { Final diagnosis } \\
\text { (OMIM phenotype } \\
\text { MIM no.) }\end{array}$ & $\begin{array}{l}\text { Material } \\
\text { change in } \\
\text { diagnosis }\end{array}$ & $\begin{array}{l}\text { Potential } \\
\text { treatment } \\
\text { change }\end{array}$ & $\begin{array}{l}\text { Nature of } \\
\text { change }\end{array}$ \\
\hline \multirow[t]{2}{*}{20} & \multirow[t]{2}{*}{2} & $20 \mathrm{~A}$ & $\begin{array}{l}\text { Cystic kidney with } \\
\text { slowly progressive } \\
\text { CKD, raised liver } \\
\text { enzymes, no evidence } \\
\text { of systemic TMA }\end{array}$ & Acute TMA & Yes & $H N F 1 B$ & $\begin{array}{l}\text { ADTKD-HNF1B } \\
(137920)\end{array}$ & Yes & Yes & $\begin{array}{l}\text { Diabetic } \\
\text { screening }\end{array}$ \\
\hline & & $20 \mathrm{~B}$ & $\begin{array}{l}\text { Congenital } \\
\text { abnormality of the } \\
\text { kidney }\end{array}$ & Oligomegonephronia & Yes & $H N F 1 B$ & $\begin{array}{l}\text { ADTKD-HNF1B } \\
(137920)\end{array}$ & Yes & Yes & $\begin{array}{l}\text { Diabetic } \\
\text { screening }\end{array}$ \\
\hline 22 & 1 & $22 \mathrm{~A}^{*}$ & $\begin{array}{l}\text { CKD diagnosed aged } \\
26, \text { hypertension, } \\
\text { father and sister with } \\
\text { history of CKD }\end{array}$ & Arteriosclerosis & Yes & WNK4 & $\begin{array}{l}\text { Pseudo- } \\
\text { hypoaldosteronism - } \\
\text { hypertensive CKD } \\
(614491)\end{array}$ & Yes & Yes & $\begin{array}{l}\text { Salt avoidance } \\
\text { and use of } \\
\text { thiazides }\end{array}$ \\
\hline 23 & 1 & $23 A^{*}$ & $\begin{array}{l}\text { Gout and progressive } \\
\text { kidney disease and } \\
\text { nephrotic range } \\
\text { proteinuria in } \\
\text { mid-20s }\end{array}$ & Severe fibrosis & Yes & SLC $3 A 1$ & $\begin{array}{l}\text { Cystinuria } \\
(220100)\end{array}$ & Yes & Yes & $\begin{array}{l}\text { Stone } \\
\text { prevention, } \\
\text { increased fluid } \\
\text { intake }\end{array}$ \\
\hline
\end{tabular}

* Genetic diagnosis as reported by [14].

NGS, next-generation sequencing; CKD, chronic kidney disease; TI, tubulointerstitial; IgA, immunoglobulin A; TMA, thrombotic microangiopathy; TBMN, thin basement membrane nephropathy; FSGS, focal segmental glomerulosclerosis; TIKD, tubulointerstitial kidney disease; GN, glomerulonephritis; DDD, dense deposit disease.

(Table 4). In the 5 cases in which a diagnosis could not be made, a family history was present in all cases.

In the chronic GN group, a genetic diagnosis was made in 4 cases (27\%; COL4A5, $n=2 ; M U C 1, n=1 ; U M O D, n=$ 1) in 4 families (Table 3). In each case, a genetic diagnosis was advanced which indicated an alternative diagnosis of kidney disease. In those in whom a COL4A5 variant was identified, one had a biopsy diagnosis of IgA nephropathy and the other a diagnosis of focal proliferative GN. In those in whom a TIKD-associated gene was identified, 1 patient $(U M O D)$ had membranoproliferative GN on biopsy. The other patient (MUC1) had a history of gout and multiple family members with kidney disease but had initially presented with a clinical as well as histological phenotype consistent with systemic lupus erythematosus (Table 4).

In the FSGS and Alport Group, genetic diagnosis was made in 6 cases (55\%) (COL4A5, $n=5$; FANCI, $n=1$;
Table 3 ) in 6 families. Four patients with an a priori diagnosis of Alport syndrome had their diagnosis confirmed (COL4A5). A further patient who had previously been simply labeled FSGS was also found to have a diagnosis of COL4A5.

In the TMA group, 10 cases (59\%) in 6 families received a genetic diagnosis (UMOD, $n=2$; $H N F 1 B, n=2$; MUC1, $n=1$; INF2, $n=4$; IFT140, $n=1$; Table 3). No patient had a phenotype consistent with a primary TMA or hemolytic uremic syndrome. In the nonspecific findings group, a genetic diagnosis was made in 6 cases (43\%; COL4A5, $n=1$; C3, $n=1$; WNK4, $n=1$; SLC3A1, $n=1$; $H N F 1 B, n=1$; INF2, $n=1$; Table 3 ). This reclassified patients with TMA or nonspecific findings into the TIKD group in 7 cases (MUC1, UMOD, IFT140, HNF1B) and into the FSGS and Alport Group in 6 cases (COL4A5, INF2 related FSGS). Three cases had nonspecific genetic 
diagnoses including pseudohypoaldosteronism (WNK4), low complement C3 (C3), and cystinuria (SLC3A1; Table $3)$.

A genetic diagnosis helped to alter or clarify the diagnosis in 31 patients (79\%) and 17 families (81\%) and materially altered the diagnosis in 21 patients $(54 \%)$ in 12 families (57\%) in whom a genetic diagnosis was made or $28 \%$ of patients and $26 \%$ of families who underwent biopsy (Table 4). A genetic diagnosis had the potential to alter treatment in 10 cases $(26 \%)$ of those with a genetic diagnosis and $13 \%$ of the total group who underwent biopsy. These potential interventions included screening, with the referral to ophthalmology and hearing assessment in 4 cases of undiagnosed Alport syndrome, diabetic screening in cases of renal cysts and diabetes syndrome, and novel treatments, such as the addition of thiazide diuretics in a patient diagnosed with pseudohypoaldosteronism (Table 4).

\section{Discussion}

Renal biopsy remains the gold standard for diagnosis of renal disease and a useful tool in predicting diagnosis and prognosis in patients with CKD. However, it remains imprecise when differentiating certain renal disorders. This is partially due to interobserver variability and partially due to heterogeneity of many kidney diseases. We have demonstrated that NGS sequencing provides a deeper understanding of the mechanism of kidney disease, and this potentially allows for more rational selection of treatment.

In our cohort, genetic diagnosis was most sensitive in TIKD. We made a diagnosis in $72 \%$ of those who had been biopsied. However, even in those groups where inherited disease is not suspected, genetic testing may be valuable. One patient diagnosed with TMA, one with MPGN and one with proliferative vasculitis were suggested to have an alternate diagnosis of familial TIKD following review. This is consistent with the findings of Groopman et al. [12] who showed that even in what are traditionally thought to be multifactorial disorders such as hypertensive or diabetic kidney disease, a monogenic diagnosis may still be identified in $1-2.5 \%$ of cases. Our findings suggest that COL4A5 disorders in adults may still be underdiagnosed on biopsy alone. This would be consistent with recent evidence that COL4A pathogenic variants are an underrecognized cause of FSGS in patients without the classic hearing loss of Alport syndrome [19, 20]. A recent paper identified monogenic disorders in $9 \%$ of adults with FSGS, the majority of which were COL4A pathogenic variants [21].

In those in which a genetic cause of kidney disease was identified, we have shown an increased precision or change in diagnosis in $81 \%$ of families and $79 \%$ of patients. This does not account for any affected family members that did not undergo biopsy, whom are also likely to be affected by genetic diagnosis. There was a potential to alter management in $26 \%$ of patients. In particular, it would allow for screening for extrarenal features, such as diabetes in patients diagnosed with diabetes and renal syndrome $(H N F 1 B)$ and hearing loss in Alport syndrome (COL4A5). Genetic diagnosis can facilitate avoidance of toxic inappropriate therapies $[22,23]$. It may help avoid corticosteroid therapy in patients with the appearance of tubulointerstitial nephritis on biopsy but a genetic diagnosis of ADTKD such as MUC1. Though none of our biopsied patients received steroids due to known family histories, many had biopsies consistent with an acute interstitial nephritis, which would traditionally receive corticosteroids.

The limitations of this study are its size. Only 39 patients had both a histological and genetic diagnosis. While care was taken to ensure a correct histological diagnosis, in a handful of cases, not all modalities were available for review, and in 2 cases, only original biopsy reports were available. In addition, it was not possible to rule out the presence of dual diagnoses. For instance, patient 7A presented with arthropathy, low C3 levels, and a biopsy showing acute GN, and they were treated acutely for systemic lupus erythematosus. While presentation of subsequent family members with CKD led to subsequent screening and detection of a pathogenic $M U C 1$ variant, the retrospective nature of the analysis means it is difficult to assess what role, if any, this played in the patient's initial presentation.

Currently, genetic testing remains time consuming and is unlikely to replace renal biopsy as the gold standard for diagnosis due to rapidity of turnaround. However, with increased availability, development of new technologies, and falling cost, we believe NGS will have a major role to play in combination with kidney biopsy in the diagnosis of CKD and may provide additional information beyond what kidney biopsy may supply.

\section{Acknowledgments}

The authors wish to acknowledge the work of Claire Foley.
Murray et al. 


\section{Disclosure Statement}

S.L.M. is funded by the Royal College of Surgeons in Ireland Hermitage Medical STAR MD, C.P.S. is supported by the Irish Research Council and Punchestown Kidney Research Fund (grant number EPSPG2015). K.A.B. is supported by the IRC Enterprise Partnership Fellowship, funded by the Irish Research Council in conjunction with the Punchestown Kidney Research fund. D.M.C. is funded by Health Research Board, Ireland (HPF-206-674), the International Paediatric Research Foundation Early Investigators' Exchange Program, and the Amgen ${ }^{\circledR}$ Irish Nephrology Society Specialist Registrar Bursary. F.H. was supported by grants from the National Institutes of Health (DK088767, DK076683, and DK068306). S.M.C. is currently supported by an academic training grant under the Irish Clinical Academic Training Programme, supported by the Wellcome Trust and the Health Research Board (Grant Number 203930/B/16/Z). Patient recruitment was funded by grants from Science Foundation Ireland (11/Y/B2093) to M.A.L., the Meath Foundation (203170.13161) to P.J.C., and the Beaumont Hospital Department of Nephrology Research Fund.

\section{Author Contributions}

S.L.M.: conception analysis and preparation of paper; A.D. and N.K.F.: review of pathology; K.A.B., D.M.C., C.P.S., K.K., G.L.C., A.J.B., and F.H.: genetic analysis; C.K.: patient recruitment and analysis; L.A.R., K.K., and M.A.L.: data collection; B.D.: paper preparation; P.J.C.: paper conception and writing.

\section{References}

1 Iversen P, Brun C. Aspiration biopsy of the kidney. Am J Med. 1951 Sep;11(3):324-30.

2 Kark RM, Muehrcke RC. Biopsy of kidney in prone position. Lancet. 1954 May;266(6821): 1047-9.

3 Racusen LC, Solez K, Colvin RB, Bonsib SM, Castro MC, Cavallo T, et al. The Banff 97 working classification of renal allograft pathology. Kidney Int. 1999 Feb;55(2):71323.

4 Weening JJ, D'Agati VD, Schwartz MM, Seshan SV, Alpers CE, Appel GB, et al.; International Society of Nephrology Working Group on the Classification of Lupus Nephritis; Renal Pathology Society Working Group on the Classification of Lupus Nephritis. The classification of glomerulonephritis in systemic lupus erythematosus revisited. Kidney Int. 2004 Feb;65(2):521-30.

5 Tøndel C, Vikse BE, Bostad L, Svarstad E. Safety and complications of percutaneous kidney biopsies in 715 children and 8573 adults in Norway 1988-2010. Clin J Am Soc Nephrol. 2012 Oct;7(10):1591-7.

6 Scheckner B, Peyser A, Rube J, Tarapore F, Frank R, Vento S, et al. Diagnostic yield of renal biopsies: a retrospective single center review. BMC Nephrol. 2009 May;10(1):11.

7 Turner MW, Hutchinson TA, Barré PE, Prichard S, Jothy S. A prospective study on the impact of the renal biopsy in clinical management. Clin Nephrol. 1986 Nov;26(5):21721.

8 Shah RP, Vathsala A, Chiang GS, Chin YM, Woo KT. The impact of percutaneous renal biopsies on clinical management. Ann Acad Med Singapore. 1993 Nov;22(6):908-11.

9 Oni L, Beresford MW, Witte D, Chatzitolios A, Sebire N, Abulaban K, et al. Inter-observer variability of the histological classification of lupus glomerulonephritis in children. Lupus. 2017 Oct;26(11):1205-11.

10 Reeve J, Sellarés J, Mengel M, Sis B, Skene A, Hidalgo L, et al. Molecular diagnosis of T cellmediated rejection in human kidney transplant biopsies. Am J Transplant. 2013 Mar; 13(3):645-55.

11 Kramer A, Pippias M, Noordzij M, Stel VS, Afentakis N, Ambühl PM, et al. The European Renal Association - European Dialysis and Transplant Association (ERA-EDTA) Registry Annual Report 2015: a summary. Clin Kidney J. 2018 Feb;11(1):108-22.

12 Groopman EE, Marasa M, Cameron-Christie S, Petrovski S, Aggarwal VS, Milo-Rasouly H, et al. Diagnostic Utility of Exome Sequencing for Kidney Disease. N Engl J Med. 2019 Jan; 380(2):142-51.

13 Muzaale AD, Massie AB, Wang MC, Montgomery RA, McBride MA, Wainright JL, et al. Risk of end-stage renal disease following live kidney donation. JAMA. 2014 Feb;311(6): 579-86.

14 Connaughton DM, Kennedy C, Shril S, Mann N, Murray SL, Williams PA, et al. Monogenic causes of chronic kidney disease in adults. Kidney Int. 2019 Apr;95(4):914-28.

15 Blumenstiel B, DeFelice M, Birsoy O, Bleyer AJ, Kmoch S, Carter TA, et al. Development and Validation of a Mass Spectrometry-Based Assay for the Molecular Diagnosis of Mucin-1 Kidney Disease. J Mol Diagn. 2016 Jul;18(4): 566-71.

16 Bleyer AJ, Hart PS, Kmoch S. Autosomal Dominant Tubulointerstitial Kidney Disease, UMOD-Related. In: Adam MP, Ardinger HH, Pagon RA, Wallace SE, Bean LJH, Stephens K, Amemiya A, editors. GeneReviews ${ }^{\circledR}$
[Internet]. Seattle (WA): University of Washington, Seattle; 1993-2019.

17 Cormican S, Connaughton DM, Kennedy C, Murray S, Živná M, Kmoch S, et al. Autosomal dominant tubulointerstitial kidney disease (ADTKD) in Ireland. Ren Fail. 2019 Nov; 41(1):832-41.

18 Richards S, Aziz N, Bale S, Bick D, Das S, Gastier-Foster J, et al.; ACMG Laboratory Quality Assurance Committee. Standards and guidelines for the interpretation of sequence variants: a joint consensus recommendation of the American College of Medical Genetics and Genomics and the Association for Molecular Pathology. Genet Med. 2015 May;17(5):405-24.

19 Varner JD, Chryst-Stangl M, Esezobor CI, Solarin A, Wu G, Lane B, et al. Genetic Testing for Steroid-Resistant-Nephrotic Syndrome in an Outbred Population. Front Pediatr. 2018 Oct; $6: 307$.

20 Malone AF, Phelan PJ, Hall G, Cetincelik U, Homstad A, Alonso AS, et al. Rare hereditary COL4A3/COL4A4 variants may be mistaken for familial focal segmental glomerulosclerosis. Kidney Int. 2014 Dec;86(6):1253-9.

21 Yao T, Udwan K, John R, Rana A, Haghighi $\mathrm{A}, \mathrm{Xu} \mathrm{L}$, et al. Integration of Genetic Testing and Pathology for the Diagnosis of Adults with FSGS. Clin J Am Soc Nephrol. 2019 Feb; 14(2):213-23.

22 Kretzler M, Cohen CD, Doran P, Henger A, Madden S, Gröne EF, et al. Repuncturing the renal biopsy: strategies for molecular diagnosis in nephrology. J Am Soc Nephrol. 2002 Jul; 13(7): 1961-72.

23 Dixon-Salazar TJ, Silhavy JL, Udpa N, et al. Exome sequencing can improve diagnosis and alter patient management. Sci Transl Med. 2012;4(138):138ra78. 